Relations industrielles

Industrial Relations

\title{
Conciliation and Arbitration Procedures in Labour Disputes.A Comparative Study, Genève, International Labour Office, 1980, 183 pp.
}

\section{Rodrigue Blouin}

Volume 36, numéro 2, 1981

URI : https://id.erudit.org/iderudit/029168ar

DOI : https://doi.org/10.7202/029168ar

Aller au sommaire du numéro

Éditeur(s)

Département des relations industrielles de l'Université Laval

ISSN

0034-379X (imprimé)

1703-8138 (numérique)

Découvrir la revue

Citer ce compte rendu

Blouin, R. (1981). Compte rendu de [Conciliation and Arbitration Procedures in Labour Disputes.A Comparative Study, Genève, International Labour Office, 1980, 183 pp.] Relations industrielles / Industrial Relations, 36(2), 453-454. https://doi.org/10.7202/029168ar

Tous droits réservés @ Département des relations industrielles de l'Université Laval, 1981
Ce document est protégé par la loi sur le droit d'auteur. L’utilisation des services d'Érudit (y compris la reproduction) est assujettie à sa politique d'utilisation que vous pouvez consulter en ligne.

https://apropos.erudit.org/fr/usagers/politique-dutilisation/ 
nourri de références recueillies ailleurs.

La bibliographie, abondante et parfaitement cosmopolite, tout en soutenant le cheminement de la pensée, est, par endroits, trop exubérante par rapport à un texte lapidaire qui aurait demandé quelques développements supplémentaires. Le discernement de l'auteur dans le choix des sources citées devrait s'exercer en vue d'un équilibre plus prononcé entre l'exposé personnel et ses références.

J'aurais également aimé qu'une fois les contours actuels dessinés, l'auteur nous entraîne quelque peu sur les voies de l'avenir (immédiat). L'éventualité d'un chômage persistant pour un nombre d'années indéterminées, l'évolution dessinée vers une économie "duale» et une gestion flexible de la maind'oeuvre, l'idée avancée d'un nouveau droit de la mobilité qui se substituerait au «garantisme» actuel, accompagnent désormais le discours-rengaine sur la destructuration du droit social. Il est vrai qu'à ce propos l'auteur ne passe pas le thème de l'«effondrement» de la législation traditionnelle du travail sous silence. Mais la vaillance dont il fait preuve, son non-conformisme salutaire auraient gagné à être suivis d'un regard lucide vers les lendemains (juridiquement) incertains qu'on nous annonce... Quels effets aura, par exemple, le télétravail sur le cadre juridique actuel?

J.-C. Javillier rappelle, par ailleurs, à propos du droit ineffectif, qui perd toute fragilité, un principe élémentaire de «marketing juridique». Ne pense-t-il pas, dans une vision large qui est la sienne, que le «marketing social» sera - est déjà - un élément déterminant des rapports sociaux (de demain)?

Edgar Morin - sociologue, mais aussi primatologue, systémiste, etc., - qui travaille aux frontières de nombreuses sciences, s'excusait dans son dernier livre, La vie de la vie, d'avoir pénétré, sans, toutefois, se les approprier, sur des territoires jalousement gardés par des spécialistes vigilants. Mair Morin est de plus en plus difficilement classable. Javillier, lui, s'il a fait un tour fécond dans des domaines connexes au sien, reste, malgré tout, fidèle à ses origines scientifiques et à sa profession. Que les juristes se rassurent: le livre dont il est question est bien celui d'un professeur de droit du travail.

Dimitri WEISS

Institut d'Administration

des Entreprises de Paris

\section{Conciliation and Arbitration Procedures in Labour Disputes. A Comparative Study, Genève, International Labour Office, $1980,183 \mathrm{pp}$.}

Cet ouvrage est d'importance. Il est en effet le premier depuis bon nombre d'années à décrire de façon aussi systématique les divers systèmes à économie libérale. Car même si l'O.I.T. a publié plusieurs études comparées sur les régimes de négociation collective, de réglementation des conflits de négociation et de résolution des mésententes juridiques, il reste que l'absence d'un ouvrage d'ensemble se faisait sentir. Le vide est comblé.

La présentation des systèmes est avant tout descriptive. Elle contient une mine de renseignements sur les sujets suivants: 1) Labour disputes and their settlement; 2) Labour relations policy and disputes settlement; 3) Formulation and implementation of national policy regarding disputes settlement; 4) Bipartite arrangements for preventing and setting disputes; 5) Governmental systems of conciliation; 6) Functioning of governmental systems of conciliation; 7) Arbitration under governmental auspices. On peut regretter que les expériences nord-américaines soient réductibles au contexte fédéral canadien et américain, privant ainsi le lecteur de données originales que l'on retrouve dans certaines provinces ou états.

Cette étude permet par ailleurs de comprendre qu'il y a une intention bien arrêtée dans la majorité des pays de trouver des modes de réglementation des conflits qui laissent toute l'initiative possible aux parties pour en arriver à une entente librement consentie.

En somme un ouvrage que ceux qui se préoccupent des expériences comparées ne pourront pas ignorer. Le texte initial d'où est 
issu cette publication a été rédigé par $\mathbf{M}$. Eladio Daya.

\section{Rodrigue BLOUIN}

Université Laval

Les grands arrêts de Droit du travail, par Gérard Lyon-Caen et Jean Pélissier, Paris, Éditions Sirey, $2^{\mathrm{e}}$ édition, 1980 , 406 pp.

La suspension du contrat de travail, par JeanMarc Béraud, Paris, Éditions Sirey, 1980, 296 pp.

International Labour Law Reports, par Zvi H. Bar-Niv (Chairman), Benjamin Aaron Thilo Ramm, Folke Schmidt, Jean-Maurice Verdier, Lord Wedderburn of Charlton (Editorial Board), Peter Elman (Coordinating Editor), Alphen aan den Rijn (PaysBas) et Germantown (Maryland, U.S.A.), volume 2, 1979, $376 \mathrm{pp}$.

Pour ceux qui, comme moi, sont des lecteurs assidus des deux éminents auteurs des Grands arrêts de Droit du Travail, cet ouvrage, dont la première édition remonte à peine à 1978 - ce qui en dit long sur son succès ne manque pas d'apparaître comme un complément nécessaire et insubstituable aux divers traités, précis et autres livres de référence qu'ils ont chacun "commis» antérieurement, séparément ou en collaboration avec d'autres.

Certes, il faut être quelque peu familier des aspects juridiques des relations industrielles pour pouvoir profiter pleinement de cette vision judiciaire du Droit du travail, rendue à travers toute une série d'arrêts de la Chambre sociale et de la Chambre criminelle de la Cour de Cassation, ainsi que du Conseil d'État français. Mais elle reste volontairement très accessible à un large public - y compris d'étudiants, qui se trouvent là à bonne école -, et ce n'est pas le moindre mérite des commentateurs que d'avoir expliqué aussi simplement, directement et sans la moindre emphase, des arrêts «grands», c'est-à-dire jugés à juste titre comme étant essentiels, rendus sur des questions non tranchées par la loi.

Par la loi française, s'entend. Est-ce à dire, par là, que ce volume s'adresse à une audience importante, certes, mais exclusivement hexagonale? Je ne le crois guère, pour peu que le lecteur ait quelque penchant pour le comparitisme. Ainsi, parmi les dix chapitres thématiques composant l'ouvrage, des «Fonctions de Droit du Travail» (Chap. I) à la «Rupture du contrat de travail» (Chap. X), qui traitent de divers aspects des rapports collectifs et individuels, le lecteur peut choisir aisément ceux qui, en fonction de ses propres intérêts et curiosités, se prêtent à un rapprochement fécond de jurisprudences diverses.

Le Canadien qui - pour rendre un cas d'actualité immédiate - peut s'interroger, par exemple, sur les conséquences juridiques de l'activité de propagande politique des syndicats dans l'entreprise (je pense à la toute récente décision arbitrale du 13 novembre 1980 ACETA/Air Canada), verrait, page 195, qu'en France, «le droit d'afficher les communications syndicales, reconnu par la loi, est interprété restrictivement (par la Cour de Cassation) afin de limiter la liberté d'affichage aux questions "professionnelles" distinctes des questions «politiques». L'employeur ne peut s'opposer lui-mêrne à l'apposition d'une affiche à contenu politique; mais il peut saisir le juge des référés qui doit ordonner l'enlèvement (...). Les mêmes règles sont transposables à la diffusion des tracts».

Plus intéressant, plus fondamental aussi - et, évidemment, plus largement exposé pour se référer à un thème central des relations industrielles, le chapitre 2 sur "Les conflits du travail», divisé en plusieurs sections et, dans sa première section sur «l'activité ouvrière», en trois grandes familles d'arrêts, respectivement sur le principe de licéité de la grève, les grèves abusives (là aussi, on débat des différences entre ce qui est "professionnel» et ce qui est «politique»), et le:s actions illicites.

Le chapitre 9 est consacré à la suspension du contrat de travail: la maladie cause de sus- 